\title{
Cold Dark Matter Substructure and the Heating of Galactic Disks
}

\author{
Andreea S. Font and Julio F. Navarro ${ }^{2}$ \\ Department of Physics and Astronomy, University of Victoria, Victoria, \\ $B C, V 8 P$ 1A1, Canada
}

\begin{abstract}
We investigate recent suggestions that substructure in cold dark matter (CDM) halos has potentially destructive effects on galactic disks. N-body simulations of disk/bulge models of the Milky Way, embedded in a dark matter halo with substructure similar to that found in cosmological simulations, show that tides from substructure halos play only a minor role in the dynamical heating of the stellar disk. This suggests that substructure might not preclude CDM halos from being acceptable hosts of thin stellar disks.
\end{abstract}

The finding of substantial amounts of substructure within virialized cold dark matter halos has brought into question the survival of thin stellar disks within such clumpy potential wells (Klypin et al 1999, Moore et al. 1999, hereafter M99). Given the abundance of galaxies with prominent, thin disk-like components, substructure poses a problem that may very well weaken the case for CDM cosmogonies and favor others, such as the warm dark matter scenario, where substructure is greatly reduced. We report here first results of a project that attempts to quantify the effects of substructure on the dynamical evolution of thin stellar disks embedded in cold dark matter halos with realistic substructure.

We have identified substructure within two galaxy-sized CDM halos; one selected from a high-resolution "standard" CDM simulation (SCDM: $\Omega_{M}=$ $1, \Omega_{\Lambda}=0, h=0.5, \sigma_{8}=0.7$ ) (see also M99 for details) and another one from a simulation of the $\Lambda \mathrm{CDM}\left(\Omega_{M}=0.3, \Omega_{\Lambda}=0.7, h=0.65, \sigma_{8}=0.9\right)$ cosmogony. The cumulative number of substructure halos, plotted in Figure 1 (left) as a function of circular velocity, appears to be roughly independent of the cosmological parameters: there is little difference in the scaled number of substructure halos in either cosmogony. Substructure halos exceed by a large factor the observed number of luminous satellites of galaxies like the Milky Way, although there is some uncertainty as to what mass scales are affected by this discrepancy. Agreement between substructure halos and luminous satellites at the high-mass end can be obtained assuming that dwarf spheroidals are surrounded by dark halos with substantially higher circular velocities than inferred from the velocity dispersion of their stars (White 2000, Font et al 2001). This still leaves a large number of substructure halos without a luminous component. Would

${ }^{2}$ CIAR Scholar and Alfred P. Sloan Research Fellow 

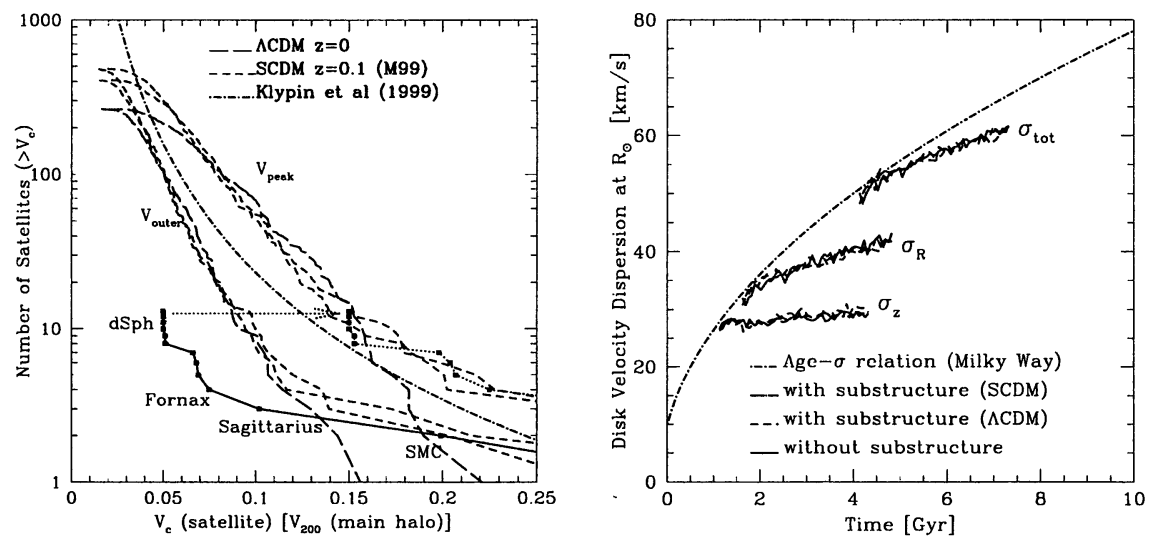

Figure 1. Left: Cumulative circular velocity function of substructure halos. Right: Stellar disk velocity dispersion as a function of time in our Milky Way model compared with the age- $\sigma_{\text {tot }}$ relation in the solar neighborhood (Fuchs et al 2000, dot-dashed curve).

these subhalos heat the thin stellar disk of the Milky Way beyond observational constraints?

We have attempted to verify this conjecture by introducing substructure halos into an equilibrium disk/bulge/halo model of the Milky Way, taking care to reproduce their masses, internal structure, characteristic densities, and orbits (Font et al 2001). For the two realizations we have analyzed so far, the global heating rate of the disk was unaffected by the addition of substructure to the dark matter halo. Indeed, even after adding substructure, the heating rate of the simulated disk is well below observational constraints (Fig. 1, right panel). This can be traced to the relatively small masses of substructure halos, typically below $10^{10}-10^{11} M_{\odot}$ in galaxy-sized halos, and to their orbits, which usually do not take them close to the disk. Our experiments suggest that previous concerns regarding excessive tidal heating of thin stellar disks by substructure in CDM halos may have been overstated.

\section{References}

Fuchs, B., Dettbarn, C., Jahreiss, H., Wielen, R. 2000, in "Dynamics of Star Clusters and the Milky Way", ASP Conference Series, eds. Deiters et al.

Klypin, A. et al, 1999, ApJ, 516, 530

Font, A., Navarro, J.F., Stadel, J., Quinn, T.R. 2001, ApJ, submitted (astro$\mathrm{ph} / 0106268)$.

Moore, B. et al, 1999, ApJ, 524, L19 (M99)

Navarro, J. F., Frenk, C. S. \& White, S. D. M. 1996, ApJ, 462, 563

White, S.D.M. 2000, "ITP Conference on Galaxy Formation and Evolution", http://online.itp.ucsb.edu/online/galaxy_c00/white/ 